\title{
Neonatal Neutrophilia: Possible Role of a Humoral Granulopoietic Factor
}

\author{
YI(IAL BARAK. "'W YORAM BI.ACHAR, AND STANLEY LEVIN
}

John A. Askin Pediatric Research Laboratory, Kaplan Hospital, Rehovot, Israel

\begin{abstract}
Summary
During the first days of life, newborn infants have leucocytosis with marked neutrophilia and a "shift to the left," the mechanism for which is as yet unknown. In an attempt to elucidate whether humoral grambepoietic factor(s) plays a role in this phenomenon, serial measurements of urinary and serum colony-stimulating activity levels were made in healthy newborn infants and normal older controls. Twenty-four-hr urine collections, serum samples, and complete blood counts were obtained from 30 full-term normal infants $24 \mathrm{hr}$ and 4 days after delivery and in 13 of them on the 14 th and 28 th days of life as well. Specimens were assayed for their colony stimulating activity levels by their ability to stimulate bone marrow cells from $\mathrm{C}_{3} \mathrm{HB}$ mice to grow into colonies in soft agar. Elevated neutrophil, band form, and monocyte counts were found during the first day of life, which gradually decreased thereafter. Serum and urinary colony-stimulating activity levels were significantly increased (3- to 5-fold) over the controls on the first and fourth days of life, but declined to normal values by the 14th and 28th days.
\end{abstract}

\section{Speculation}

The rise in neutrophils and band forms in the newborns is caused by stimulation of granulopoiesis which is regulated by colony stimulation activity, and which probably occurs during the late stage of pregnancy and the first postnatal days. The possibility that production of these elevated colony stimulation activity levels is either by the newborn's monocytes or the placenta remains to be determined.

Marked neutrophilia is known to occur in newborn infants during the first days of life. At the same time there is a significant increase in the numbers of band forms, metamyelocytes, and monocytes. This initial peak with the exception of monocytes gradually falls towards the end of the first wk of life when the lymphocyte becomes the predominant cell $(8,14)$. The mechanism for this phenomenon is obscure: neither granulocytic overproduction nor cell displacement from storage or marginal pools has been proved to be the cause.

In the last decade, a method for short-term marrow culture in soft agar has been developed which enabled the identification of an in vitro granulopoietic factor with colony-stimulating activity (CSA) in the serum and urine of animals and men $(10,11)$. Human CSA can be assayed with mouse bone marrow cells as target cells (13). In the present study, the levels of urinary and serum CSA in full-term newborns were serially assayed to determine the role of this humoral granulopoietic factor in neonatal neutrophilia.

\section{MATERIALS AND METHODS}

Studies were made on 30 healthy full-term newborns, with informed parental consent. Complete blood counts, serum samples, and 24-hr urine collections were obtained from all newborns within $12 \mathrm{hr}$ of birth and on the fourth day of life and from 13 of them on the 14th and 28 th days as well. Twenty-four-hr urine collections and serum samples were also obtained from 10 healthy adults who have served as normal controls.

Urinary and serum CSA levels were determined by modifications of the methods of Robinson $(10,11)$. In brief, $0.1 \mathrm{ml}$ of dialyzed serum or $0.15 \mathrm{ml}$ of dialyzed sedimented filter-sterilized urine was placed in triplicate $35-\mathrm{mm}$ plastic Petri dishes (Nunclon). To this was added a mixture of $0.3 \%$ agar in McCoy's $5 \mathrm{~A}$ medium containing $10^{5}$ nucleated bone marrow cells from the femurs of $\mathrm{C} 3 \mathrm{H} / \mathrm{bl}$ mice. The plates were then incubated at $37^{\circ} \mathrm{C}$ in a fully humidified atmosphere containing $10 \%$ CO.2. Colony counts were performed after 7 days as an indicator of the level of granulopoietic activity in the specimen. Only colonies containing 50 or more cells were counted. Previous data have shown a linear relationship between the number of such colonies and the amount of granulopoietic activity present, allowing quantitation of the CSA present in the serum or urine sample (13). The CSA activity was expressed as the mean number of colonies from triplicate plates per $0.15 \mathrm{ml}$ of $24-\mathrm{hr}$ urine or per $0.1 \mathrm{ml}$ of serum $(10.11)$.

\section{RESULTS}

Table 1 presents the results of total neutrophil, band form, and monocyte counts of the entire study group. In agreement with previous data $(8,14)$, neutrophilia with "shift to the left" was prominent on the first day of life and decreased gradually thereafter. Monocytosis was pronounced during the first 2 wk and decreased by about $50 \%$ by 4 wk of life. Also, a significant number of metamyelocytes and a few myelocytes could be observed in most newborns on the first day of life.

Figure 1 shows the mean levels of urinary CSA on the first. fourth. 14th. and 28th days of life as compared with the levels of normal adults urinary CSA in our laboratory. At the ages of 1 and 4 days, mean urinary CSA levels were $49 \pm 27$ and $32 \pm 21$ colonies per $0.15 \mathrm{ml}$, respectively, compared to the normal values of $9 \pm 8$ colonies per $0.15 \mathrm{ml}(P<0.001)$. By the $14 \mathrm{th}$ and $28 \mathrm{th}$ days, mean CSA levels decreased almost to normal $(12 \pm 10$ and $11 \pm 8$ colonies per $0.15 \mathrm{ml}$, respectively).

In Figure 2, the individual pattern of urinary CSA levels in each of the 13 newborns who were followed serially for $4 \mathrm{wk}$ are presented. Almost uniformly, there were peak CSA levels in the first $24 \mathrm{hr}$, decreasing somewhat by the fourth day, and dropping significantly at the age of 14 and 28 days.

Figure 3 shows the mean levels of serum CSA of newborns as compared with the normal adult serum CSA levels in our laboratory. On the first and fourth days of life, mean serum CSA levels were $26 \pm 16$ and $21 \pm 12$ colonies per $0.1 \mathrm{ml}$, respectively, again showing a highly signifcant increase in CSA as compared with the normal values of $5 \pm 4$ colonies per $0.1 \mathrm{ml}(P<0.001)$. On the 14 th and 28th days, mean serum CSA levels dropped to $15 \pm 12$ colonies per $0.1 \mathrm{ml}$, still higher than in the controls but the difference being statistically insignificant $(P>0.02)$. A significant correlation between urinary and serum CSA levels and neutrophil, band, and monocyte counts was not demonstrated. This is not 
Table 1. Summary of absolute neutrophil, band-form, and monocyte counts in the first month of newborn life

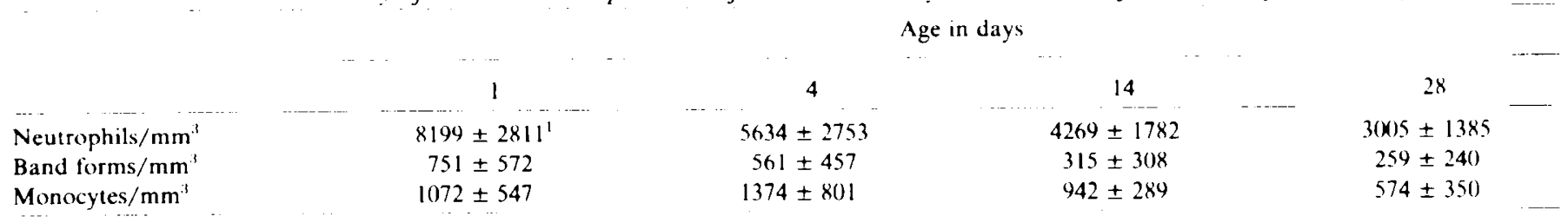

Mean \pm S. D

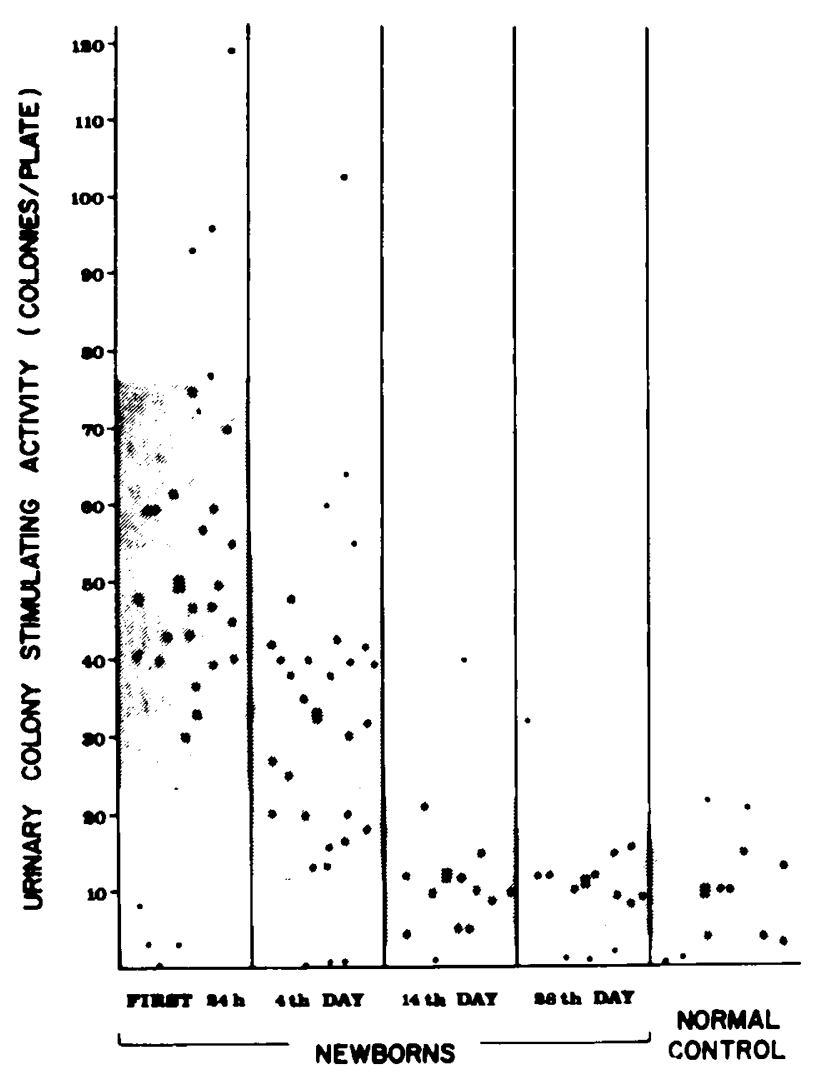

Fig. 1. Urinary ( $S A$ levels in 30 full-term newborns during the first 4 wh of life. $\overline{\mathbf{x}}$, mean; shaded areas, $\pm \mathrm{S} . \mathrm{D}$.

unexpected because diurnal fluctuations known to occur in leukocyte counts of the newborn could account for this lack of correlaton.

\section{DISCUSSION}

Theoretically, the postnatal elevation in neutrophils, band forms, and monocytes in the peripheral blood, which were also found in the present study, could be attributed to one of several mechanisms. These include granulocyte overproduction, release of eclls from granulocytic marrow reserves or from the marginating pool, and hemoconcentration. Inasmuch as this neutrophilia includes a simultaneous rise in bands and monocytes with a decrease in the number of lymphocytes (8), it seems unlikely that hemoconcentration could be the true explanation. Xanthou (14) favors the "displacement" theory rather than overproduction as the most likely cause because in her studies she found only a small rise in neutrophil precursors in newborn blood, however, certain features of neonatal neutrophilia point to an increased granulocyte production as the most likely mechanism. Most workers in this field have noted the marked shift to the left. increased numbers of metamyelocytes, myelocytes, promyelocytes, and even blasts in the peripheral blood during the first postnatal days (8). Bone marrow aspirates during the first wk of life shows marked cellularity, with increased numbers of granulocytic precursors (12). At

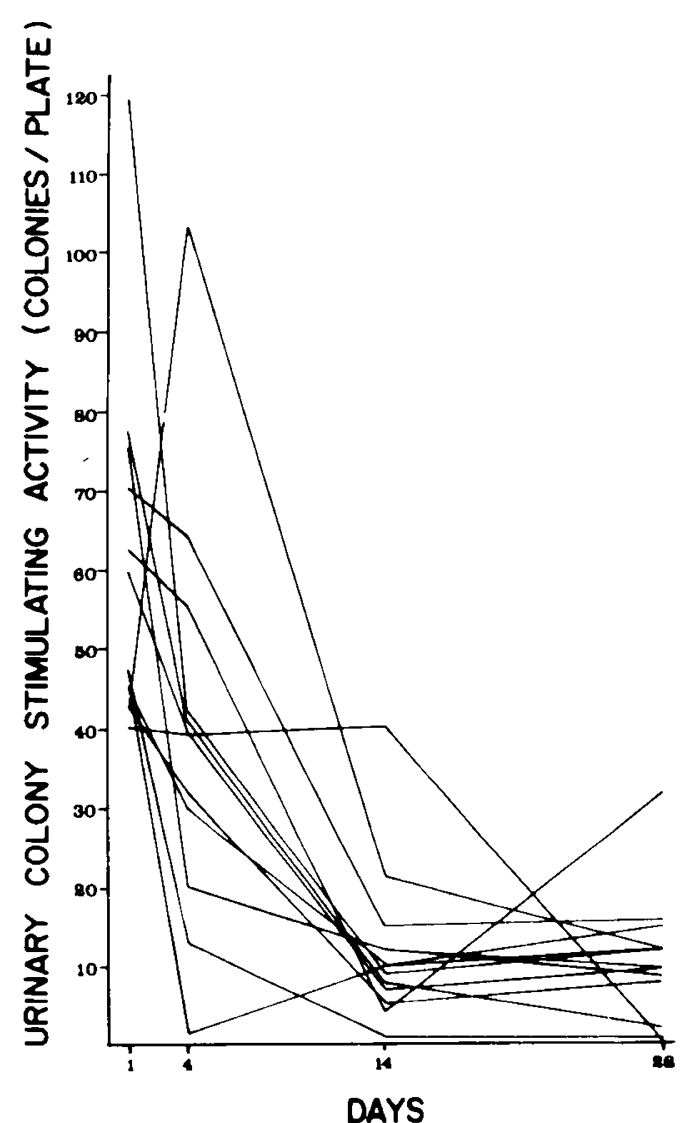

Fig. 2. Serial urinary (SA determinations in 13 newborns. Lines individual patients

the same time, the 4 days duration of the neonatal neutrophil rise appears to be too long to be a release or displacement phenomenon, which regularly takes a matter of hours (1).

The results of the present study demonstrate a significant elevation of granulocytic CSA in the urine and serum of normalterm newborns during the first 4 days of life, together with increased neutrophil counts. Several lines of evidence, such as CSA injections in mice leading to neutrophilia (7) and the demonstrations in CSA levels corresponding to neutrophil counts in various neutropenias (5). strongly suggest that CSA might be a true in vivo granulopoietic factor. It is suggested. therefore, that a humoral factor regulating granulocytic proliferation might be the main cause for the production of neutrophilia in the newborns.

The finding of elevated CSA levels along with a marked neutrophilia during the first wk of life was not unexpected. Apart from previous observation indicating a very active granulopoiesis in the bone marrow and peripheral blood of the newborn infant during the first wk of life $(8)$, recent studies $(6,9)$ have demonstrated an increased concentrations of granulocytic colony-forming cells in cord blood. indicating an expansion and proliferation of granulocyte committed stem cells in the newborn. The cell or cells which are involved in the production of increased CSA in 


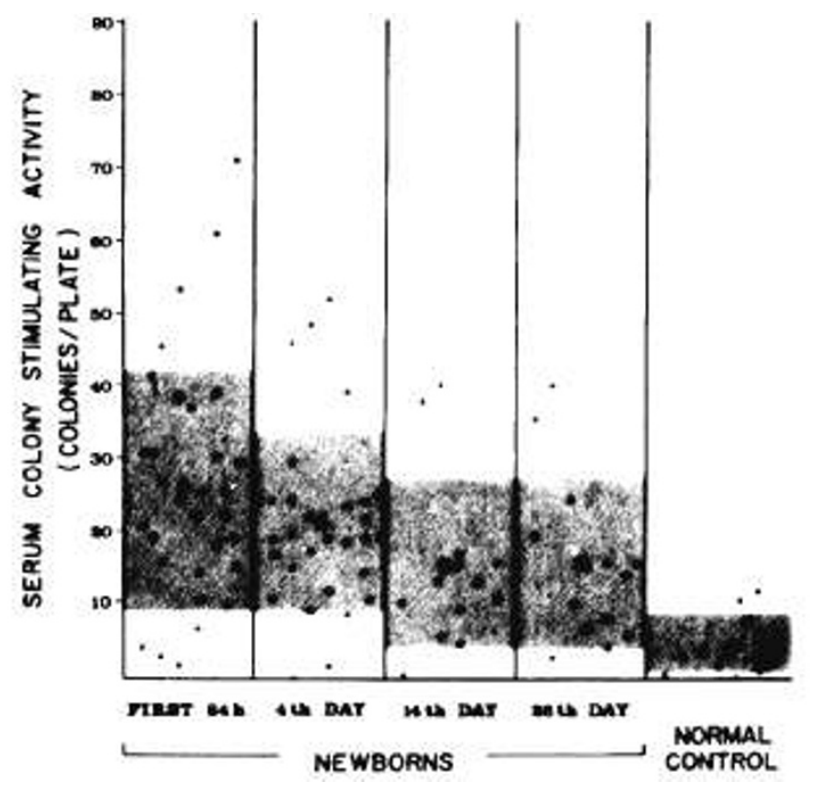

Fig. 3. Serum CSA levels in 30 full-term newborns during the first 4 wk of life. $\bar{x}$, mean; shaded areas, \pm S.D.

the newborn are unknown, and this important fact remains to be elucidated. However, it has been shown that monocytes are the main source of CSA in man (3). Inasmuch as monocytosis has been consistently found during the neonatal period $(8,14)$, the possibility that monocytes are the source of the increased CSA levels must be raised. However, another fascinating finding has been recently reported, indicating that human placenta (2) is a very potent source of granulocytic CSA. It remains to be determined by further studies involving pregnant women and their babies whether placental CSA is related to the neutrophilia com- monly found in pregnant women at term (4) and to the neutrophilia of the newborn infants.

\section{REFERENCES AND NOTES}

I. Boggs, D. R.: Physiology of neutrophil proliferation, maturation and circulation. Clin. Haematol.. 4: 535 (1975).

2. Burgess, A. W., Wilson, E. M. A., and Metcalf. D.: Stimulation by human placental conditioned medium of hemopoietic colony formation by human marrow cells. Blood. 49: 573 (1977).

3. Chervenick. P. A., and Lobuglio, A. F.: Human blood monocytes: stimulators of granulocyte and mononuclear colony formation in vitro. Science II (Wash. D. (.). 178: 164 (1972).

4. Efrati. P.. Presentey, B., Margalith, M., and Rozenszajn, L.: Leukxcytes of normal pregnant women. Obstet. Gynecol., 23: 429 (1964).

5. Greenberg. P. L., and Schrier, S. L.: Granulopoiesis in neutropenic disorders. Blood, 41: 753 (1973)

6. Knudtzon, $S$.: In vitro growth of granulocytic colonies from circulating cells in human cord blood. Blood, 43: 357 (1974)

7. Metcalf, D., and Stanley, E. R.: Haematological effects on mice of partially purified colony stimulating factor (CSF) prepared from human urine. $\mathrm{Br}$. $\mathrm{J}$. Haematol.. 21: 481 (1971)

8. Oski, F. A., and Naiman, J. L.: Normal blood values in the newborn period. In Hematologic problems in the newborn. pp I-30 (W. B. Saunders, Philadelphia. 1972)

9. Prindull, G., Prindull, B., and Meulen, N. V. D.: Hematopoietic stem cells (CFUC) in human cord blood. Acta Paediatr. Scand., 67: 413 (1978).

10. Robinson, W. A., Bradley. T. R., and Foster, R.: Bone marrow colony stimulating activity in human sera. Br. J. Haematol., 15: 147 (1968)

11. Robinson, W. A., and Pike, B. L.: Leukopoietic activity in human urine. N. Engl J. Med.. 282: 1291 (1970).

12. Shapiro. L. M., and Bassen. F. A.: Sternal marrow changes during the first week of life. Correlation with peripheral blood findings. Am. J. Med. Sci., 20?: 341 (1941).

13. Stanley, E. R.. and Metcalf, D.: Partial purification and some properties of the factor in normal and leukemic human urine stimulating mouse bone marrow colony growth in vitro. Aust. J. Exp. Biol. Med. Sci., 47: 467 (1969)

14. Xanthou, $M$.: Leukocyte blood picture in health full-term and premature babies during neonatal period. Arch. Dis. Child., 45: 242 (1970).

15. Requests for reprints should be addressed to: Yigal Barak, M.D. Pediatric Research Laboratory, Kaplan Hospital, P. O. Box I, Rehovot, Israel.

16. This research was supported by a grant from the Chief Scientist's Office. Israel Ministry of Health.

17. Received for publication August 27, 1979.

18. Accepted for publication November 13, 1979 